\title{
ON LINEARIZED THEORY FOR COMPRESSIBLE VISCOUS PLANAR FLOW WITH SLIP*
}

\author{
$\mathrm{By}$ \\ F. E. FENDELL \\ TRW Systems, Redondo Beach, Calif.
}

\begin{abstract}
Steady compressible supersonic planar flow of a viscous heat-conducting fluid is examined under linearization about freestream conditions. Although the results are derived here for a large-Prandtl-number fluid, $\mathrm{Wu}$ has shown that the conclusions hold for an order-unity-Prandtl-number fluid in the limits of large and small Reynolds numbers. First the compressible correction to the fundamental solution for flow past a singular flat plate (a point source of momentum directed antiparallel to the uniform freestreaming) is examined. Results valid near the point source are obtained by contour integration. Then these results are superposed to form an integral equation describing linearized slip flow past a finite flat plate at zero angle of attack. The large-slip limit for a short plate is characterized as a regular perturbation limit while the small-slip limit has singular-perturbation characteristics. For a long flat plate the integral equation can be approximated as Poisson type and is easily solved; for a short flat plate the integral equation can be approximated as a Carleman equation of the second kind and is less readily solved. However, an approximate solution for the shear near the leading edge of a short flat plate with small slip is given through the Wiener-Hopf technique. The integral equation describing thermal slip at a finite flat plate is seen to be like that describing velocity slip.

1. Introduction. Steady planar supersonic flow is here examined for Reynolds numbers so low that there is no preferred direction for diffusion. The continuum model is postulated to be valid for such conditions. However, such conditions are too rarefied for adequate treatment by conventional merged-layer asymptotic analysis and a different attack is required. For tractability the viscous compressible flow equations, linearized about freestream conditions in the manner of Oseen [1], are taken to be uniformly valid. Except near stagnation points, anticipated slip in both temperature and tangential velocity at a solid boundary suggests small deviation from uniform freestream conditions [2]. The usual reason for sharp gradients near the body, the no-slip boundary conditions, is absent.
\end{abstract}

Much work has recently been done on linearized viscous incompressible flow past lifting source distributions acting at arbitrary angles to the freestreaming [3, and the references cited therein]. Here attention is confined to sources acting parallel or antiparallel to the freestreaming [4]-[10]. The emphasis on compressibility, slip, and finite body size is novel. Linearized results are suspect in the transonic range [4, p. 94].

The solution for an individual source of mass, momentum, or energy in an unbounded

*Received December 11, 1968. Support of this work by TRW Systems under its Independent Research and Development Program and by the U. S. Army Research Office (Durham, North Carolina) under contact DAHCO-67-C-0015 is gratefully acknowledged. 
expanse of fluid is here referred to as a fundamental solution [4]. In principle, because of the linearization, one can superpose fundamental solutions to constitute any arbitrary but small disturbance to the uniform freestream. Further, the fundamental solution itself may be expressed as the superposition of a solenoidal (transverse) wave and an irrotational (longitudinal) wave. The transverse wave is the same as in incompressible flow and concerns the transport of vorticity. There are no pressure, density, or temperature disturbances associated with this wave. Such disturbances are governed by the equations of viscous heat-conducting acoustics, which describe the longitudinal wave. A velocity potential and a force potential exist for the longitudinal wave, so that the dependent variables occurring in the equations for this wave may be expressed entirely in terms of scalar quantities. Still further decomposition of a fundamental solution is sometimes possible. For example, the irrotational part of the transverse wave may be isolated from the rotational part, or the compressible and incompressible contributions to the longitudinal wave may be separated.

A concise review of linearized theory as developed by Lagerstrom and others is given in Secs. 2 and 3. Explicit formulae for the contribution due to fluid compressibility for flow past a point source of momentum directed antiparallel to the freestream are given for the first time for the region near the source in Sec. 4. Characteristics of the integral equation formed by superposing point sources to simulate slip flow past a flat plate at zero angle of attack are discussed in Sec. 5. The integral equation is approximately solved for the following special cases: for a long plate by Laplace transform techniques (Sec. 6), a short plate with moderate slip by Picard iteration (Sec. 7), and for a short plate with slight slip by Wiener-Hopf techniques (Sec. 8). The extraction of previously derived no-slip results from the current solutions with slip is discussed in each case. Comparison is also made with other solutions for slip flow given for either linear or nonlinear models. While the results are explicitly derived for velocity slip in a large-Prandtl-number fluid, their validity for an order-unity-Prandtl-number fluid and their straightforward extension for describing thermal slip is indicated in Sec. 9.

2. Governing equations. Nondimensionalization of the dependent variables against their freestream values and the independent variables against a viscous length $\left(\mu_{\infty}^{*} / \rho_{\infty}^{*} q_{\infty}^{*}\right)$ yields in the conventional notation:

$$
\begin{gathered}
p=\rho T, \quad \nabla \cdot(\rho \mathbf{q})=0, \\
\rho \mathbf{q} \cdot \nabla \mathbf{q}=-\frac{1}{\gamma \mathrm{M}^{2}} \nabla p+\epsilon \mathbf{X}+\nabla \cdot(\mu \operatorname{def} \mathbf{q})+\nabla \cdot[(\nabla \cdot \mathbf{q}) \zeta \mathrm{I}], \\
\rho \mathbf{q} \cdot \nabla T-\frac{\gamma-1}{\gamma} \mathbf{q} \cdot \nabla p=\frac{1}{\operatorname{Pr}} \nabla \cdot(k \nabla T)+\frac{\mathrm{M}^{2}(\gamma-1)}{2}\left[\mu(\text { def } \mathbf{q})^{2}+\zeta(\nabla \cdot \mathbf{q})^{2}\right]+\epsilon Q .
\end{gathered}
$$

Here $\operatorname{Pr}$ and $M$ are the freestream Prandtl and Mach numbers, respectively ( $c_{p}^{*}$ const.). The symmetric tensor def $\mathrm{q}=\nabla \mathrm{q}+(\nabla \mathrm{q})^{T}$ where superscript $T$ denotes transpose. $\mathbf{X}$ is the perturbational force per unit volume nondimensionalized against $\left(\rho_{\infty}^{*^{2}} q_{\infty}^{*^{3}} / \mu_{\infty}^{*}\right)$ and $Q$, the perturbational heat source per volume-time nondimensionalized against $\left(\rho_{\infty}^{* 2} q_{\infty}^{* 2} c_{p}^{*} T_{\infty}^{*} / \mu_{\infty}^{*}\right)$. The magnitude of the disturbance is of order $\epsilon$, where now $0<\epsilon \ll 1$. Far from the body (if $\hat{\imath}$ is the freestreaming direction)

$$
\mathrm{q} \rightarrow \hat{\mathbf{1}}, \quad p \rightarrow 1, \quad T \rightarrow 1 .
$$

Slip conditions on the body will be formulated later. 
For small disturbances, $\mathrm{q}=\hat{\mathbf{i}}+\epsilon \mathrm{q}^{\prime}, \rho=1+\epsilon s, p=1+\epsilon p^{\prime}, T=1+\epsilon T^{\prime}, \mu=$ $1+\epsilon \mu^{\prime}, k=1+\epsilon k^{\prime}, \zeta=\zeta_{\infty}+\epsilon \zeta^{\prime}$. Substituting into Eqs. (2.1)-(2.4) and dropping the primes,

$$
\begin{gathered}
p=s+T, \quad \partial s / \partial x+\nabla \cdot \mathbf{q}=0, \\
\frac{\partial \mathbf{q}}{\partial x}=-\frac{1}{\gamma \mathrm{M}^{2}} \nabla p+\mathbf{X}-\nabla x(\nabla x \mathbf{q})+\left(2+\zeta_{\infty}\right) \nabla(\nabla \cdot \mathbf{q}), \\
\frac{\partial}{\partial x}\left(T-\frac{\gamma-1}{\gamma} p\right)=\frac{1}{\operatorname{Pr}} \nabla^{2} T+Q,
\end{gathered}
$$

subject to the conditions far from the body

$$
q \rightarrow 0, \quad p \rightarrow 0, \quad T \rightarrow 0 .
$$

The coordinate $x$ is measured parallel to the freestreaming. There has been no need to specify the dependence of the transport properties on the thermodynamic state. The dissipation term has been omitted because quantities of $O\left[\epsilon^{2} \mathrm{M}^{2}(\gamma-1)\right]$ are taken as uniformly negligible. This approximation is responsible for the relative unimportance of momentum responses to thermal sources and of thermal responses to momentum sources in cases to be discussed below. Finally, the Stokes relation $\zeta_{\infty}=-2 / 3$ is adopted for explicitness; this relation holds for monatomic gases only and results may be readily derived for other values of $\zeta_{\infty}$ if experimental data are available.

3. The singular flat plate in a large-Prandtl-number fluid without heat sources. For $Q=0, \operatorname{Pr} \gg 1$ one recovers, by using the boundary conditions at infinity, the isentropic relationship between the thermodynamic variables [4]:

$$
T=(\gamma-1) / \gamma p=>p=\gamma s .
$$

One need just deal thenceforth with the continuity equation and momentum equations. Since $\operatorname{Pr}=O(1)$ for gases, this approximation may seem incompatible with the retention of compressibility effects. The reconciliation has been given by $\mathrm{Wu}[6]$ and will be discussed below.

Here the fundamental solution for planar two-dimensional flow is sought for the so-called singular flat plate (a shear force of unit strength located at the origin in an unbounded expanse of fluid and acting on the fluid in the direction opposite to the freestreaming):

$$
\mathrm{X}=-\delta(x) \delta(y) \hat{\mathbf{1}} .
$$

If the velocity perturbation for this point source is denoted $-\boldsymbol{\Gamma}(x, y)$ and the condensation perturbation $-s(x, y)$, the solution for any distribution of forces $X_{x}(x, y)$ acting in the same direction is

$$
\begin{aligned}
q_{x}(x, y) & =\iint_{-\infty}^{\infty} \Gamma_{x}(x-\xi, y-\eta) X_{x}(\xi, \eta) d \xi d \eta, \\
q_{y}(x, y) & =\iint_{-\infty}^{\infty} \Gamma_{y}(x-\xi, y-\eta) X_{x}(\xi ; \eta) d \xi d \eta, \\
s(x, y) & =\iint_{-\infty}^{\infty} S(x-\xi, y-\eta) X_{x}(\xi, \eta) d \xi d \eta
\end{aligned}
$$


Fourier transform and inversion yields $[4]\left[r=\left(x^{2}+y^{2}\right)^{1 / 2}\right]$ :

$$
-\boldsymbol{\Gamma}_{1}=\sum_{i=1}^{4} \mathbf{q}_{i}
$$

where

$\mathbf{q}_{1}=\frac{1}{2 \pi} \quad \nabla\left[\exp (x / 2) K_{0}(r / 2)\right]=$ irrotational part of transverse wave,

$\mathbf{q}_{2}=-\frac{1}{2 \pi} \exp (x / 2) K_{0}(r / 2) \hat{\imath}=$ rotational part of transverse wave,

$\mathbf{q}_{3 \bar{i}}=\frac{1}{2 \pi} \quad \nabla(\ln r)=$ incompressible part of longitudinal wave,

$\mathbf{q}_{4}=$ compressible correction to the longitudinal wave.

Previously the method of Laplace [11] has given an approximate expression for $\mathrm{q}_{4}$ for large $y$ in both the supersonic and subsonic cases [4], [6]. Here the contribution of $\mathbf{q}_{4}$ for small $y$ is undertaken.

4. The compressible contribution to the fundamental solution for a singular fiat plate. If $a=3 / 4 \mathrm{M}^{2}$ and $b=3\left(1-\mathrm{M}^{2}\right) / 4 \mathrm{M}^{2}$ then [4]

$$
\begin{aligned}
& -\mathrm{q}_{4}=\left\{\begin{array}{l}
-q_{4_{x}} \\
-q_{4_{v}}
\end{array}\right\} \\
& =\frac{1}{4 \pi i} \int_{-\infty}^{\infty} \frac{\exp (i \beta x)}{\beta}\left\{\begin{array}{c}
-\beta^{2} \\
i \beta \frac{\partial}{\partial y}
\end{array}\right\}\left[\frac{(\beta-a i)^{1 / 2}}{\left(\beta^{2}\right)^{1 / 2} \frac{{ }^{1 / 2}}{(\beta-b i)^{1 / 2}} \exp \left(-|y| \frac{\left(\beta^{2}\right)^{1 / 2}(\beta-b i)^{1 / 2}}{(\beta-a i)^{1 / 2}}\right)}\right. \\
& \left.\quad-\frac{\exp \left(-\left(\beta^{2}\right)^{1 / 2}|y|\right)}{\left(\beta^{2}\right)^{1 / 2}}\right] d \beta, \\
& s=-S=-\frac{\mathrm{M}^{2}}{4 \pi} \int_{-\infty}^{\infty} \exp (i \beta x)\left[\frac{(\beta-a i)^{1 / 2}}{(\beta-b i)\left(\beta^{2}\right)^{1 / 2}} \exp \left(-|y| \frac{\left(\beta^{2}\right)^{1 / 2}(\beta-b i)^{1 / 2}}{(\beta-a i)^{1 / 2}}\right)\right] d \beta .
\end{aligned}
$$

The definition of $\left(\beta^{2}\right)^{1 / 2}$ is such that the real part of the argument of the exponents remains negative definite for all $y$. Thus, in the second term in the square brackets in Eq. (4.1) $\left(\beta^{2}\right)^{1 / 2}=|\beta|=\beta(\operatorname{sgn} \beta)$; however, the first term in the square brackets is more subtle. For $1>M>0\left(\mathrm{q}_{4}=0\right.$ for $\mathrm{M}=0$ by construction), there are two branch points on the positive imaginary axis, one at $\beta=a i$ and one at $\beta=b i$. Here $a$ and $b$ are real and positive, and $a>b$. As $\mathrm{M} \uparrow 1$ the branch point at $b i$ goes to the origin, and moves down the negative imaginary axis for $M>1$. As $M \rightarrow \infty$, the branch point at ai descends toward the origin along the positive imaginary axis, while the branch point in the lower-half plane goes to $-3 i / 4$. For $M>1$ (the case of interest here) the correct choice for the first term in the square brackets in Eq. (4.1) is $\left(\beta^{2}\right)^{1 / 2}=\beta$; the choice insures that the real part of $\left(\beta^{2}\right)^{1 / 2}(\beta-b i)^{1 / 2} /(\beta-a i)^{1 / 2}$ remains positive. (The branches of the square roots are chosen to be such that the expressions are real and positive for large positive real $\beta$, a choice adopted throughout this discussion.) The branch cuts for $M>1$ are chosen as in Fig. 1 . 


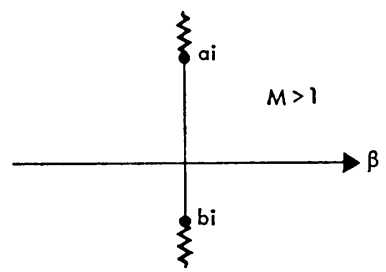

FIG. 1. Choice of branch cuts in the $\beta$ plane for supersonic freestreaming.

Although explicit results will not be sought for $\mathrm{M}<1$, it is noted that $(\beta-a i)^{1 / 2} /(\beta-b i)^{1 / 2}$ has a positive real part everywhere on the real axis in subsonic flow. For a negative definite argument for the exponential function, one must define $\left(\beta^{2}\right)^{1 / 2}=|\beta|=\beta(\operatorname{sgn} \beta)$ in the first term in square brackets. However, $|\beta|$ is not an analytic function, and a common ploy is to let

$$
\sqrt{\beta^{2}} \rightarrow\left(\beta^{2}-\delta^{2}\right)^{1 / 2}=(\beta+i \delta)^{1 / 2}(\beta-i \delta)^{1 / 2}
$$

where $\delta$ is a small positive real number. The limiting process $\delta \rightarrow 0$ is deferred until a convenient point in the analysis, and direct substitution justifies the procedure. The branch cuts are taken as in Fig. 2.

With these conventions one may write for the supersonic case

$$
-\mathrm{q}_{4}=\left\{\begin{array}{c}
-q_{4 x} \\
-q_{4 y}
\end{array}\right\}=\left\{\begin{array}{l}
I_{1}+I_{3} \\
I_{2}+I_{4}
\end{array}\right\}
$$

where

$$
\begin{aligned}
& I_{1}=-\frac{1}{4 \pi i} \int_{-\infty}^{\infty}\left(\frac{\beta-a i}{\beta-b i}\right)^{1 / 2} \exp \left[i \beta x-|y| \frac{\beta(\beta-b i)^{1 / 2}}{(\beta-a i)^{1 / 2}}\right] d \beta, \\
& I_{2}=-\frac{\operatorname{sgn} y}{4 \pi} \int_{-\infty}^{\infty} \exp \left[i \beta x-|y| \beta\left(\frac{\beta-b i}{\beta-a i}\right)^{1 / 2}\right] d \beta, \\
& I_{3}=\frac{1}{4 \pi i} \int_{-\infty}^{\infty}(\operatorname{sgn} \beta) \exp [i \beta x-|y||\beta|] d \beta=\frac{1}{2 \pi} \frac{x}{x^{2}}+y^{2} \\
& I_{4}=\frac{\operatorname{sgn} y}{4 \pi} \int_{-\infty}^{\infty} \exp [i \beta x-|\beta||y|] d \beta=\frac{1}{2 \pi} \frac{y}{x^{2}+y^{2}} .
\end{aligned}
$$

By Jordan's lemma the paths of integration are now deformed as shown in Fig. 3; these paths suggest letting $\beta=i \alpha$.

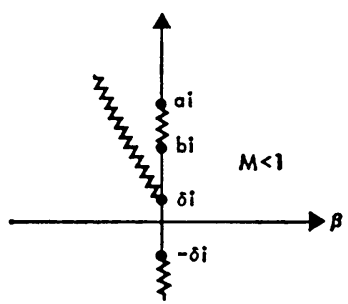

Frg. 2. Branch cuts in the $\beta$ plane for the subsonic case. 


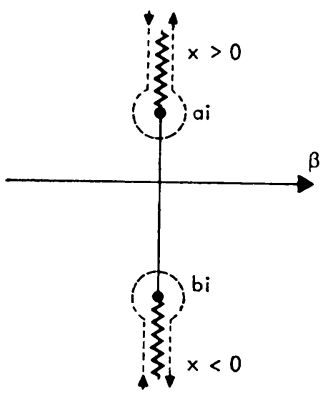

FIG. 3. Contour paths for the compressibility-correction integrals.

The following well-known Laplace transform will be useful below [12], [13]:

$$
J=\int_{0}^{\infty} \frac{\exp (-\gamma x)}{\left[\gamma\left(\gamma+a^{\prime}\right)\right]^{1 / 2}}=K_{0}\left(a^{\prime} x / 2\right) \exp \left(a^{\prime} x / 2\right) \quad a^{\prime}>0, \quad x>0 .
$$

By differentiation

$$
\int_{0}^{\infty}\left(\frac{\gamma}{\gamma+a^{\prime}}\right)^{1 / 2} \exp (-\gamma x) d x=\frac{a^{\prime}}{2}\left[K_{1}\left(a^{\prime} x / 2\right)-K_{0}\left(a^{\prime} x / 2\right)\right] \exp \left(a^{\prime} x / 2\right) .
$$

Also required will be

$I=\int_{0}^{\infty}\left(\frac{\sigma+a^{\prime}}{\sigma}\right)^{1 / 2} \exp (-\sigma x) d \sigma=\exp \left(a^{\prime} x\right) \int_{-a^{\prime}}^{\infty}\left(\frac{y}{y-a^{\prime}}\right)^{1 / 2} \exp (-y x) d y$.

But

$$
I=a^{\prime} J-\frac{d J}{d x}=\frac{a^{\prime}}{2} \exp \left(\frac{a^{\prime} x}{2}\right)\left[K_{0}\left(a^{\prime} x / 2\right)+K_{1}\left(a^{\prime} x / 2\right)\right] .
$$

Differentiation of $I$ and $J$, together with recurrence relations for the modified Bessel functions of the second kind, permits one to evaluate all the real integrals that will arise in connection with $\mathbf{q}_{4}$ for small $y$.

For supersonic flow for $x>0$ integration of integral $I_{1}$ around the contour shown in Fig. 3 gives

$$
-4 \pi I_{1}=2 \int_{a}^{\infty}\left(\frac{\alpha-a}{\alpha-b}\right)^{1 / 2} \cos \left[\alpha|y|\left(\frac{\alpha-b}{\alpha-a}\right)^{1 / 2}\right] \exp (-\gamma x) d \alpha
$$

Letting $\gamma=\alpha-a$, noting $a-b=3 / 4$, expanding for small $y$, and invoking Eq. (4.10):

$$
I_{1}=\frac{3}{16 \pi}\left\{\exp \left[\frac{3\left(\mathrm{M}^{2}-2\right) x}{8 \mathrm{M}^{2}}\right]\right\}\left\{K_{1}\left(\frac{3 x}{8}\right)-K_{0}\left(\frac{3 x}{8}\right)\right\}+O\left(y^{2}\right) \quad \mathrm{M}>1, \quad x>0 .
$$

For supersonic flow for $x<0$

$$
-4 \pi I_{1}=2 \int_{-|b|}^{-\infty}\left(\frac{\alpha-a}{\alpha-|b|}\right)^{1 / 2} e^{\alpha|x|}\left[\cos \alpha|y|\left(\frac{\alpha+|b|}{\alpha-a}\right)^{1 / 2}\right] d \alpha .
$$

Letting $\sigma=-\alpha-|b|$, noting $a+|b|=3 / 4$, expanding for small $y$, and invoking Eq. $(4.12)$

$$
I_{1}=\frac{3}{16 \pi}\left\{\exp \left[\frac{3\left(2-\mathrm{M}^{2}\right)}{8 \mathrm{M}^{2}}|x|\right]\right\}\left\{K_{0}\left(\frac{3|x|}{8}\right)+K_{1}\left(\frac{3|x|}{8}\right)\right\}+O\left(y^{2}\right) \quad \mathrm{M}>1, x<0 .
$$


Similarly for $\mathrm{M}>1, x>0$

$$
I_{2}=-\frac{\operatorname{sgn} y}{2 \pi} e^{-a x} \int_{0}^{\infty} e^{-\tau x} \sin \left[\left(\frac{\tau+3 / 4}{\tau}\right)^{1 / 2}(\tau+a)|y|\right] d \tau .
$$

For small $y$

$$
\begin{aligned}
I_{2}=-\frac{9 y}{64 \pi}\left\{\exp \left[\frac{3 x}{\mathrm{M}^{2}}\left(\mathrm{M}^{2}-2\right)\right]\right\} & \left\{\left(\frac{2-\mathrm{M}^{2}}{2 \mathrm{M}^{2}}\right) K_{0}\left(\frac{3 x}{8}\right)\right. \\
+ & \left.\left(\frac{1}{\mathrm{M}^{2}}-\frac{4}{3 x}\right) K_{1}\left(\frac{3 x}{8}\right)+\frac{1}{2} K_{2}\left(\frac{3 x}{8}\right)\right\}+O\left(y^{3}\right) .
\end{aligned}
$$

Finally, for $\mathrm{M}>1, x<0$

$$
I_{2}=-\frac{\operatorname{sgn} y}{2 \pi} e^{-|b||x|} \int_{0}^{\infty} e^{-\sigma|x|} \sin \left[(\sigma+|b|)\left(\frac{\sigma}{\sigma+\frac{3}{4}}\right)^{1 / 2}|y|\right] d \sigma .
$$

For small $y$

$$
\begin{aligned}
I_{2}=-\frac{3 y}{16 \pi}\left\{\exp \left[\frac{3\left(2-\mathrm{M}^{2}\right)}{8 \mathrm{M}^{2}}|x|\right]\right\} & \left\{\left(\frac{6-3 \mathrm{M}^{2}}{8 \mathrm{M}^{2}}\right) K_{0}\left(\frac{3|x|}{8}\right)\right. \\
- & {\left.\left[K_{1}\left(\frac{3|x|}{8}\right)\right]\left[\frac{3}{4 \mathrm{M}^{2}}+\frac{1}{|x|}\right]+\frac{3}{8} K_{2}\left(\frac{3|x|}{8}\right)\right\} . }
\end{aligned}
$$

Addition of the four contributions to the velocity field for compressible viscous flow past a singular flat plate yields for all $y$ for $M>1$

$$
\begin{aligned}
& q_{x}(x, y)=-I_{1}(x, y)-\frac{e^{x / 2}}{4 \pi}\left[K_{0}(r / 2)+\frac{x}{r} K_{1}(r / 2)\right], \\
& q_{\nu}(x, y)=-I_{2}(x, y)-\frac{e^{x / 2}}{4 \pi} \frac{y}{r} K_{1}(r / 2) .
\end{aligned}
$$

Explicit use will be made of the result

$$
q_{x}(x, 0)=-I_{1}(x, 0)-\frac{e^{x / 2}}{4 \pi}\left[K_{0}\left(\frac{|x|}{2}\right)+(\operatorname{sgn} x) K_{1}\left(\frac{|x|}{2}\right)\right]
$$

where

$$
\begin{aligned}
I_{1}(x, 0) & =\frac{3}{16 \pi}\left\{\exp \left[\frac{3\left(\mathrm{M}^{2}-2\right) x}{8 \mathrm{M}^{2}}\right]\right\}\left\{-K_{1}\left(\frac{3 x}{8}\right)+K_{0}\left(\frac{3 x}{8}\right)\right\} \quad x>0, \\
& =\frac{3}{16 \pi}\left\{\exp \left[\frac{3\left(2-\mathrm{M}^{2}\right)|x|}{8 \mathrm{M}^{2}}\right]\right\}\left\{K_{1}\left(\frac{3|x|}{8}\right)+K_{0}\left(\frac{3|x|}{8}\right)\right\} \quad x<0 .
\end{aligned}
$$

Recalling asymptotic forms, one finds that as $x \downarrow 0$ or $x \uparrow 0$ for M $>1$ from Eqs. (4.23) and $(4.24)\left(\gamma_{1}=\right.$ Euler's constant $\left.=0.57721 \ldots\right)$ :

$q_{x}(x, 0)=-\Gamma_{x}(x, 0)=\frac{1}{16 \pi}\left\{7 \ln \frac{|x|}{4}+\left[\left(7 \gamma_{1}-4\right)+3\left(\frac{\mathrm{M}^{2}-2}{\mathrm{M}^{2}}\right)+3 \ln \frac{3}{4}\right]\right\}$.

This is, of course, functionally the same source-like form as found for the incompressible case [10]

$$
q_{x}(x, 0)=-\Gamma_{x}(x, 0)=\frac{1}{4 \pi}\left\{\ln \frac{|x|}{4}+\left[\gamma_{1}-1\right]\right\} .
$$


For $y=0, x \rightarrow \infty$ from Eqs. (4.23) and (4.24)

$$
q_{x}(x, 0)=-\Gamma_{x}(x, 0) \sim-(4 \pi x)^{-1 / 2}
$$

as in the incompressible case. (The compressible correction contained in $I_{1}$ is exponentially small as $x \rightarrow \infty$ for all finite supersonic Mach numbers.) For $y=0, x \rightarrow-\infty$, for $M>1$

$$
q_{x}(x, 0)=-\Gamma_{x}(x, 0) \sim \frac{\exp (-|x|)}{4 \pi|x|^{3 / 2}}-\left(\frac{3}{16 \pi|x|}\right)^{1 / 2} \exp \left[\frac{3}{4}\left(\frac{1-M^{2}}{M^{2}}\right)|x|\right] .
$$

The compressible correction dies off less rapidly upstream than the incompressible portion for all finite Mach numbers exceeding unity, but the decay is still exponential.

5. Extension to a finite flat plate with slip. Superposition permits extension of the singular flat plate to a plate of finite length positioned at zero angle of attack to the freestream (say, of dimensional length $2 a^{*}$, with midchord at the origin). If the boundary condition for slip is taken in the usual linear form [14], [15], then on the plate

$$
1+\epsilon q_{x}(x, 0)=\lambda \epsilon\left|\frac{\partial q_{x}(x, 0)}{\partial x}\right|, \quad|x| \leq \operatorname{Re} \equiv \frac{a^{*}}{\left(\mu_{\infty}^{*} / \rho_{\infty}^{*} q_{\infty}^{*}\right)}
$$

where $\lambda$, the dimensionless slip parameter, is [Kn $(2-\sigma) / \sigma]$. Here $\operatorname{Kn}$ is the Knudsen number (the ratio of the molecular mean free path to the typical diffusive length characterizing the flow) and $\sigma$ is the Maxwell reflection coefficient for the plate surface. Since $\sigma=1$ for a perfectly diffuse surface, and many surfaces behave nearly so, often $\lambda \doteqdot \mathrm{Kn}$. The linear slip relation, once thought to hold for small slip only, now is commonly adopted for all magnitudes of slip. As $\lambda \rightarrow 0$ (no slip), the inadequacy of the linearization becomes apparent because the small perturbation must cancel the freestreaming. Therefore, the linearization is formally extended to $\epsilon=1$; such an approximation is inherent in all linearized studies of flows past bodies in which inertia terms must be retained (cf. Enskog's procedure for the Boltzmann equation according to Chapman and Cowling [16]).

Since the two-dimensional force distribution

$$
X_{x}(x, y)=g(x) H(\operatorname{Re}-|x|) \delta(y)
$$

for a plate lying on $y=0,|x|<\operatorname{Re}$ and since $\Gamma_{x}(x, y)$ was given for a negative point source, the slip boundary condition may be rewritten as

$$
1+\int_{-\mathrm{Re}}^{\mathrm{Re}} \Gamma_{x}(x-\xi, 0) g(\xi) d \xi=-\frac{\lambda}{2} g(x), \quad|x| \leq \mathrm{Re} .
$$

This linear inhomogeneous Fredholm integral equation of the second kind with displacement kernel [17] was derived on the basis that the force associated with a flat plate at zero angle of attack is all due to the shear on the plate; the factor $(1 / 2)$ arises because the force $g(x)$ is the shear acting on both sides of the flat plate, top and bottom. The above integral equation was apparently first stated for incompressible slip flow by Laurmann [14]; here the proper kernel $\Gamma_{x}(x, 0)$ for compressible supersonic slip flow has been explicitly given. The slip velocity along the plate is given directly by the right-hand side of the equation, $-\lambda g(x) / 2$. Finally, it is noted that one can associate a homogeneous integral equation with the inhomogeneous one by letting

$$
\int_{-\mathbf{R}_{\mathbf{0}}}^{\mathrm{Re}_{\mathbf{0}}}\left[1+\Gamma_{x}(x-\xi, 0)\right] h(\xi) d \xi=-\frac{\lambda}{2} h(x), \quad g(x)=h(x) / \int_{-\mathbf{R}_{\mathbf{0}}}^{\mathrm{Re}} h(v) d v .
$$


For $\lambda \rightarrow \infty$ (perfect slip), $g \rightarrow 0$; for $\lambda \gg 1$ iteration will converge to the solution, as described below. This parametric limit has the features of a regular perturbation expansion. For the opposite limit $\lambda=0$ linearized theory is known to give a squareroot singularity for the shear; yet for any finite $\lambda$, no matter how small, linearized theory should give a finite shear at the leading and trailing edges on physical grounds. For $0<\lambda \ll 1$ one anticipates that $g(x ; \lambda=0)$ is substantially modified only for $x \simeq \pm$ Re. Such boundary-layer behavior suggests that the limit $\lambda \rightarrow 0$ is singular.

6. Approximate solution for a long flat plate with slip. From Eqs. (4.26) and (4.27) $q_{x}$ vanishes exponentially rapidly for $x \ll 0$ for a source at the origin, but decays only as the inverse square-root of the distance from the source for $x \gg 0$. For a long flat plate lying on $y=0,0 \leq x \leq \operatorname{Re}$ where $\operatorname{Re} \gg 1$ the kernel is approximated [4]

$$
-\Gamma_{x}(x, 0)=q_{x}(x, 0)=-\frac{1}{(4 \pi x)^{1 / 2}} H(x) .
$$

The error incurred by the inaccurate representation near $x=0$ might seem indeterminate, but the results will reveal the nature of the approximation. Use of this kernel in Eq. (5.3) yields a Poisson integral equation:

$$
1+\frac{1}{(4 \pi)^{1 / 2}} \int_{0}^{x} \frac{g(\xi)}{(x-\xi)^{1 / 2}} d \xi=-\frac{\lambda}{2} g(x), \quad 0 \leq x \leq \text { Re. }
$$

For $\lambda=0$ (no slip) one recovers the Abel integral [17] equation formulated and solved in [4]. The absence of the plate length Re or of the Mach number $M$ is to be noted. Kinetic theory suggests that $\mathrm{M}=O(\lambda)$. Application of the Laplace transform yields:

$$
g(x)=-\frac{1}{\lambda \pi i} \int_{c-i \infty}^{c+i \infty} \frac{e^{s x}}{s^{1 / 2}\left(s^{1 / 2}+\lambda^{-1}\right)} d s
$$

or

$$
g(x)=-\frac{2}{\lambda} e^{x / \lambda^{2}} \operatorname{erfc}\left(x^{1 / 2} / \lambda\right) .
$$

This is the same result given by Mirels [18] and later by Bell [19] by treating the linearized partial differential equations governing compressible viscous flow past a semiinfinite flat plate in the boundary-layer approximation (retention of diffusion normal to the plate only). The kernel approximation is apparently equivalent to the boundarylayer approximation. Asymptotic forms of (6.4) are (the small $x$ result is of particularly dubious validity since changes over distances shorter than a mean free path are being described):

$$
g(x) \sim-\frac{2}{\lambda}\left[1+\left(\frac{1}{\lambda^{2}}-\frac{2}{\pi^{1 / 2}}\right) x+O\left(x^{2}\right)\right], \quad \frac{x^{1 / 2}}{\lambda} \rightarrow 0 .
$$

[The shear $(-g)$ increases away from the leading edge if $.94>\lambda$.]

$$
g(x) \sim \frac{-2}{(\pi x)^{1 / 2}}\left[1-\frac{\lambda^{2}}{2 x}+O\left(\frac{\lambda^{4}}{x^{2}}\right)\right], \quad \frac{x^{1 / 2}}{\lambda} \rightarrow \infty .
$$

If $D^{*}$ is the drag per unit span, then the nondimensional force on the plate in the direction of the freestream (the drag coefficient) is

$$
C_{D}=\frac{D^{*}}{\rho_{\infty}^{*} q_{\infty}^{* 2} a^{*}}=-\frac{1}{\operatorname{Re}} \int_{0}^{\mathrm{Re}} g(x) d x .
$$


Equations (6.4) and (6.7) give

$$
C_{D}=\frac{4 \lambda}{\operatorname{Re}}\left\{\frac{1}{2} \exp \left(\operatorname{Re} / \lambda^{2}\right) \operatorname{erfc}\left(\operatorname{Re}^{1 / 2} / \lambda\right)-\frac{1}{2}+\frac{\operatorname{Re}^{1 / 2}}{\pi^{1 / 2} \lambda}\right\} .
$$

Asymptotic forms are

$$
\begin{gathered}
C_{D} \sim \frac{4}{(\pi \mathrm{Re})^{1 / 2}}\left[1-\frac{\pi^{1 / 2} \lambda}{2 \operatorname{Re}^{1 / 2}}+O\left(\lambda^{2}\right)\right], \quad \lambda \rightarrow 0, \\
C_{D} \sim \frac{2}{\lambda^{2}}+O\left(\lambda^{-3}\right), \quad \lambda \rightarrow \infty .
\end{gathered}
$$

Laurmann [14] studied steady linearized viscous incompressible flow past a semiinfinite flat plate with slip via the Wiener-Hopf technique. He solved Eq. (5.3) with modified integral limits and a kernel composed of $-\left[q_{1 s}(x, 0)+q_{2_{x}}(x, 0)+q_{3_{z}}(x, 0)\right]$. He found for $x \rightarrow \infty$, in contrast to (6.6),

$$
g(x) \sim-\frac{2}{(\pi x)^{1 / 2}}\left\{1+\frac{\lambda}{2 \pi x}\left[\ln \left(\frac{x}{2}\right)-0.04\right]-\frac{\lambda^{2}}{2 x}+\cdots\right\} .
$$

For any finite slip, Laurmann finds that far from the leading edge the full linearized equation gives a different shear from that predicted on the basis of the boundary layer approximation; in fact, for all $x \gg 1$ obeying $x>2 \exp [0.04+\pi \lambda]$, Laurmann states that the presence of slip increases the magnitude of the shear at the plate.

The question of what the correct results are for an Oseen model of slip flow past a long flat plate is academic (except possibly for finding higher-order corrections) if the Oseen model is not a reasonable approximation to the full nonlinear Navier-Stokes equations. Murray [20] studied the steady viscous incompressible flow past a semiinfinite flat plate at zero angle of attack under the usual linear slip relation. Using the full nonlinear equations, Murray found for $\left(x^{1 / 2} / \lambda\right) \gg 1$

$$
g(x) \sim-\frac{1.32824}{2 x^{1 / 2}}\left[1+\nu^{\prime} \frac{\lambda^{2}}{x} \ln \left(\frac{\lambda}{x^{1 / 2}}\right)+\gamma^{\prime} \frac{\lambda^{2}}{x}+\cdots\right] .
$$

Because $\nu^{\prime}$ and $\gamma^{\prime}$ remain undetermined constants, Murray states that his results (which are different from those obtained from use of the Oseen equations) are tentative: the validity of his conclusions depend on what solution is eventually found to hold closer to the leading edge. However, even if Eq. (6.12) is valid, one should not conclude that the use of the Oseen equations is not appropriate for a study of slip phenomena. Since slip is of diminishing importance as $x$ increases, and the linear model is particularly suspect near the plate when slip is vanishingly small, discrepancies between the linear (Oseen) model and the nonlinear equations are not surprising far from the leading edge. Where slip is appreciable, the Oseen equations may very well be valid approximations.

7. Approximate solution for a short flat plate with moderate slip. For a short flat plate lying on $y=0,-\operatorname{Re} \leq x \leq \operatorname{Re}$ where Re $\ll 1$, Eqs. (4.25), (4.26), and (5.3) show

$$
1+\int_{-R \mathbf{e}}^{R \mathrm{e}}(\alpha+\beta \ln |x-\xi|) g(\xi) d \xi=-\frac{\lambda}{2} g(x), \quad|x| \leq \operatorname{Re}
$$

where

$$
M=0: \alpha=-\left(\gamma_{1}-1-\ln 4\right) / 4 \pi, \beta=-1 / 4 \pi,
$$


$M>1: \alpha=-\left[7 \gamma_{1}-4+3\left(M^{2}-2\right) / M^{2}+3 \ln (3 / 4)-7 \ln 4\right] / 16 \pi, \beta=-7 / 16 \pi$. . (7.3)

The solution of Eq. (7.1) is known to be even in $x$ so similar behavior is anticipated at the leading and trailing edges of the short plate.

For $\lambda=0$ solution of (7.1) was given by Carleman [11] and others:

$$
g(x)=\frac{-1}{\pi[\alpha+\beta \ln (\operatorname{Re} / 2)]} \frac{1}{\left(\operatorname{Re}^{2}-x^{2}\right)^{1 / 2}} .
$$

Eq. (7.4) was given first by Bairstow, Cave, and Lenz [4] by a different approach. Despite the singularities in the shear at the plate extremities, the drag is clearly finite:

$$
C_{D}=-\frac{1}{2 \operatorname{Re}} \int_{-R \mathrm{e}}^{R \mathrm{e}} g(x) d x=\frac{1}{2[\alpha+\beta \ln (\mathrm{Re} / 2)] \operatorname{Re}} .
$$

Finite slip involves treating the inhomogeneous linear Fredholm equation of the second kind with symmetric displacement kernel of logarithmic type, Eq. (7.1). For large enough slip approximate solution is achieved by Picard iteration (or, equivalently, regular perturbation in descending power of $\lambda$ ). If $p(x)=\lambda g(x) / 2$

$$
-p_{n+1}(x)=1+\mu^{\prime} \int_{-R \mathrm{e}}^{R \mathrm{e}}(\alpha+\beta \ln |x-\xi|) p_{n}(\xi) d \xi
$$

where $\mu^{\prime}=2 / \lambda$ and $p_{0}(x)=-1$ by choice. Since

$$
\int_{-R \mathrm{e}}^{R \mathrm{e}}(\alpha+\beta \ln |x-\xi|)^{2} d \xi \leq C_{1}, \text { a const. }
$$

the successive approximations $p_{n}(x)$ converge to the unique solution provided [17]

$$
\mu^{\prime}\left\{\int_{-R \Theta}^{R \Theta} d x \int_{-R \Theta}^{R \Theta}[\alpha+\beta \ln |x-\xi|]^{2} d \xi\right\}^{1 / 2} \leq 1
$$

or

$\mu^{\prime} \operatorname{Re} 2^{1 / 2}\left\{2 \alpha^{2}-6 \alpha \beta+7 \beta^{2}+2 \beta(2 \alpha-3 \beta) \ln (2 \operatorname{Re})+2 \beta^{2} \ln [(2 \operatorname{Re})]^{2}\right\}^{1 / 2} \leq 1$.

Substitution of $\alpha$ and $\beta$ reveals that Eq. (7.9) is satisfied for $\lambda \geq O(1)$ for all $M$. Since $M=O(\lambda)$, only the supersonic values of $\alpha$ and $\beta$ may be justifiably used. But even then the results of iteration are of uncertain validity because $(\operatorname{Re} / \lambda) \sim[$ (plate length)/ (mean free path)] $<O(1)$. That is, continuum theory is being used to describe changes occurring over a fraction of a mean free path. Nevertheless, it is interesting to proceed purely formally. Iteration shows that the presence of slip leads to a finite shear at the plate ends:

$$
\begin{aligned}
&-g(x) \simeq-g_{1}(x)=\mu^{\prime}+\mu^{\prime 2}\{2 \operatorname{Re}(\alpha-\beta)+\beta[(x+\operatorname{Re}) \ln (x+\mathrm{Re}) \\
&+(\operatorname{Re}-x) \ln (\operatorname{Re}-x)]\} \\
& C_{D}=-\frac{1}{2 \operatorname{Re}} \int_{-R \mathrm{e}}^{R \mathrm{e}} g(x) d x=\frac{2}{\lambda}\left\{1-\frac{2 \operatorname{Re}}{\lambda}[(2 \alpha-3 \beta)+2 \beta \ln (2 \mathrm{Re})]\right\} .
\end{aligned}
$$

The lowest-order result in Eq. (7.10) is invariant with Mach number and plate length and has been given by Laurmann [2]. The lowest-order invariance recalls the leading term in Eq. (6.5). A possible physical interpretation of the constant shear to 
lowest order is that no freestream molecule intersecting the flat plate has first undergone a collision with a molecule that has already collided with the plate.

The full expression of (7.10) gives $-g$ even about its maximum, which occurs at midchord $(x=0)$; the magnitude of the difference between maximum and minimum values of $g$ is $2 \ln 2|\beta| \mu^{\prime 2}$ Re. For Re $<1$ [Eq. (7.9) holds only for $\operatorname{Re}<1$ ], decreasing the slip coefficient $\lambda$ increases the shear $(-g)$ and thus the $\operatorname{drag} C_{D}$.

Numerical evaluation of Eq. (7.10) for Re $=.2$ with $\lambda=1$ or 5 is given in Fig. 4 for several Mach numbers. Since $\lambda=O(M)$, the $M=0$ results are given merely for contrast. All cases plotted satisfy Eq. (7.9). Monte-Carlo simulation on a digital computer of colliding molecules in a supersonic rarefied gas flowing past a flat plate at zero angle of attack is being conducted at TRW Systems by G. Bird, G. Broadwell, and F. Vogenitz and will be reported in the near future. These calculations show the shear gradually increasing to a midchord peak before decreasing (like the shear distribution shown in Fig. 4) for cases of Maxwellian ("soft") molecules or plate lengths not very much greater than the mean free path. Furthermore, the calculations give the magnitude of the shear coefficient $(-g)$ to be $O\left(10^{-1}\right)$. Since the Monte Carlo calculations are probably on a firm basis and the present continuum model is not, the similarities may be fortuitous.

The criterion (7.8) is not fulfilled for small enough $\lambda$ for a fixed Re, and attention is now turned to this case.

8. Approximate solution for the shear at the leading edge of a short flat plate with small slip. The Oseen model is less accurate for the slight slip limit than for the moderate

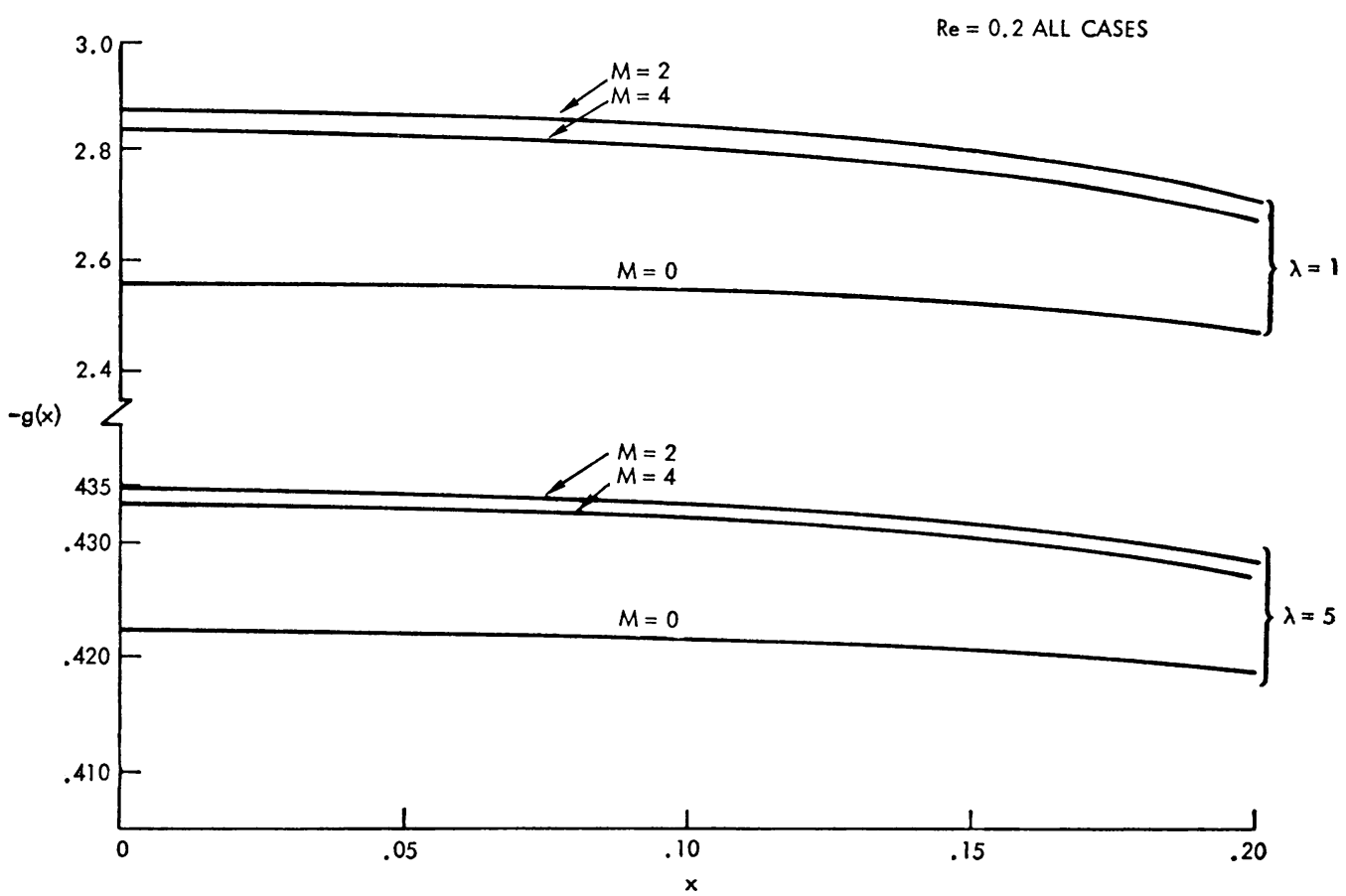

FIG. 4. The shear distribution predicted by Eq. (7.10). 
slip case just treated. Nevertheless, the singular small-slip limit retains some physical (as well as appreciable mathematical) interest. Because $\mathrm{M}=0, \alpha$ and $\beta$ should be restricted to their $M=0$ values in this section.

To solve Eq. (7.1) for $\lambda \ll 1$, one may consider

$$
A+\frac{\beta^{*}}{\pi} \int_{-\mathrm{Re}}^{\mathrm{Re}_{\mathrm{e}}} K_{0}\left[\beta^{*}|x-t|\right] g(t) d t=-\lambda^{*} g(x)
$$

where $A, \beta^{*}$, and $\lambda^{*}$ are constants to be determined. Since $\operatorname{Re} \ll 1$, the kernel may be expanded to give

$$
\frac{A}{Q \beta^{*}}+\frac{1}{Q \pi} \int_{-\mathrm{Re}}^{\mathrm{Re}}\left[\ln \left(2 / \beta^{*}\right)-\gamma_{1}-\ln |x-t|\right] g(t) d t \simeq-\frac{\lambda^{*}}{Q \beta^{*}} g(x)
$$

where $Q$ is another constant. Equations (7.1) and (8.2) are equivalent if

$$
A=Q \beta^{*}, \lambda^{*}=Q \beta^{*} \lambda / 2, Q \pi \beta=-1, \alpha Q \pi=\ln \left(2 / \beta^{*}\right)-\gamma_{1} .
$$

Letting $h(t)=g(t) / A, T=(t / \operatorname{Re})+1, z=(x / \operatorname{Re})+1$, and $\beta^{\prime}=\operatorname{Re} \beta^{*}$,

$$
1+\frac{\beta^{\prime}}{\pi} \int_{0}^{2} K_{0}\left[\beta^{\prime}|z-T|\right] H\left(T^{\prime}\right) d T=-\lambda^{*} H(z)
$$

where $h[\operatorname{Re}(T-1)] \rightarrow H(T)$. It is recalled that for $\lambda^{*}=0$, if the kernel is expanded as in Eq. (8.2), one obtains [cf. Eq. (7.4)]

$$
H(z)=\left\{\beta^{\prime}\left[\gamma_{1}+\ln \left(\beta^{\prime} / 4\right)\right]\right\}^{-1}[z(2-z)]^{-1 / 2} .
$$

Substituting Eq. (8.4) in Eq. (8.3) shows that, for $\lambda^{*} \rightarrow 0$, Eq. (8.4) is inadequate near $z=0$ and $z=2$ only.

An equation that is tractable by the Wiener-Hopf technique [11] is

$$
1+\frac{\beta^{\prime}}{\pi} \int_{0}^{\infty} K_{0}\left(\beta^{\prime}|z-T|\right) H(T) d T=-\lambda^{*} H(z) .
$$

This equation recovers the behavior of (8.4) as $z \downarrow 0$ to within a multiplicative factor for $\lambda^{*}=0$ (demonstrated below), and it should be a good approximation to Eq. (8.3) for $z \downarrow 0$ for $\lambda^{*} \ll 1$.

If one defines $H(z)=0$ for $z<0$, defines $g(z)=-1$ for $z>0$ and $g(z)=0$ for $z<0$, and introduces $p(z)$ where $p(z)=0$ for $z>0$, then Eq. (8.5) may be written

$$
\frac{\beta^{\prime}}{\pi} \int_{-\infty}^{\infty} K_{0}\left(\beta^{\prime}|z-T|\right) H(T) d T=g(z)+p(z)-\lambda^{*} H(z) .
$$

The Fourier transform and inversion is here defined:

$$
g(\xi)=\int_{-\infty}^{\infty} \exp (-i \xi z) g(z) d z, \quad g(z)=\frac{1}{2 \pi} \int_{-\infty}^{\infty} \exp (i \xi z) g(\xi) d \xi .
$$

Fourier transformation of Eq. (8.6) under convolution is

$$
\frac{\left(\xi+i \beta^{\prime}\right)_{+}^{1 / 2}}{i \xi_{-}}+\frac{\beta^{\prime}+\lambda^{*}\left({\beta^{\prime}}^{2}+\xi^{2}\right)^{1 / 2}}{\left(\xi-i \beta^{\prime}\right)_{-}} H(\xi)_{-}=\left(\xi+i \beta^{\prime}\right)_{+}^{1 / 2} p(\xi)_{+}
$$

where the plus subscript denotes a function analytic in the upper-half $\xi$ plane and the negative subscript, a function analytic in the lower-half plane. Since $\beta^{\prime}+\lambda^{*}\left(\beta^{\prime 2}+\xi^{2}\right)^{1 / 2}$ 
has branch points at $\xi= \pm i \beta^{\prime}$ and no other singularities, a strip of common analyticity lying in $-i \beta^{\prime}<\operatorname{Im}(\xi)<0$ is anticipated. Eq. (8.8) is rewritten

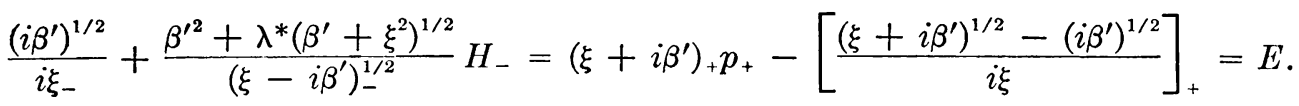

$E$ is an entire function. Since $H\left(z \rightarrow 0, \lambda^{*}=0\right) \sim z^{-1 / 2}$ and $H\left(z \rightarrow 0, \lambda^{*} \ll 1\right)$ is bounded, one anticipates $E=0$.

For $\lambda^{*}=0$, inversion immediately gives [12, p. 57, pair 549]

$$
H(z)=-\left\{\left(\pi \beta^{\prime} z\right)^{-1 / 2} \exp \left(-\beta^{\prime} z\right)+\operatorname{erf}\left(\beta^{\prime} z\right)^{1 / 2}\right\} .
$$

Comparing Eqs. (8.4) and (8.10) shows that for $z \rightarrow 0$ shear on a short plate exceeds that on a long plate; the freestreaming past a semi-infinite flat plate must be faster for the leading-edge shear to be raised to the short-flat-plate level. The behavior of (8.10) at large $z$ is of less interest.

For $\lambda^{*} \neq 0$, if $\xi^{\prime}=\xi / \beta^{\prime}$, standard splitting techniques [11] yield

$$
K\left(\xi^{\prime}\right)=1+\lambda^{*}\left(\xi^{\prime 2}+1\right)^{1 / 2}=\frac{L\left(\xi^{\prime}\right)_{+}}{M\left(\xi^{\prime}\right)_{-}}=\frac{\exp \left[-\frac{1}{2 \lambda^{*}} \int^{\xi^{\prime}} R(x)_{+} d x\right]}{\exp \left[+\frac{1}{2 \lambda^{*}} \int^{\xi^{\prime}} S(x)_{-} d x\right]} .
$$

If $k=\left(1-\lambda^{*^{2}}\right)^{1 / 2} / \lambda^{*}$,

$$
\begin{aligned}
R\left(\xi^{\prime}\right)_{+}= & \left(\xi^{\prime}-k\right)^{-1}\left\{\frac{(\pi / 2)+i \ln \left[\xi^{\prime}+\left(\xi^{\prime 2}+1\right)^{1 / 2}\right]}{\pi\left(\xi^{\prime 2}+1\right)^{1 / 2}}-\frac{(\pi / 2)+i \ln \left(\lambda^{*-1}+k\right)}{\pi / \lambda^{*}}\right\} \\
& +\left(\xi^{\prime}+k\right)^{-1}\left\{\frac{(\pi / 2)+i \ln \left[\xi^{\prime}+\left(\xi^{2}+1\right)^{1 / 2}\right]}{\pi\left(\xi^{\prime 2}+1\right)^{1 / 2}}-\frac{(\pi / 2)+i \ln \left(\lambda^{*-1}-k\right)}{\pi / \lambda^{*}}\right\}, \\
S\left(\xi^{\prime}\right)_{-}= & \left(\xi^{\prime}-k\right)^{-1}\left\{\frac{(\pi / 2)-i \ln \left[\xi^{\prime}+\left(\xi^{\prime 2}+1\right)^{1 / 2}\right]}{\pi\left(\xi^{\prime 2}+1\right)^{1 / 2}}-\frac{(\pi / 2)-i \ln \left(k+\lambda^{*-1}\right)}{\pi / \lambda^{*}}\right\} \\
& +\left(\xi^{\prime}+k\right)^{-1}\left\{\frac{(\pi / 2)-i \ln \left[\xi^{\prime}+\left(\xi^{\prime 2}+1\right)^{1 / 2}\right]}{\pi\left(\xi^{\prime 2}+1\right)^{1 / 2}}-\frac{(\pi / 2)-i \ln \left(\lambda^{*-1}-k\right)}{\pi / \lambda^{*}}\right\} .
\end{aligned}
$$

Since Eq. (8.11) states $\left(1+\lambda^{*}\right) \mathrm{M}(0)_{-}=L(0)_{+}$, Eq. (8.9) is formally rewritten

$$
\begin{aligned}
\frac{\left(i \beta^{\prime}\right)^{1 / 2}}{\left(1+\lambda^{*}\right)[\mathrm{M}(0)]_{-} i \xi_{-}} & +\frac{\beta^{\prime} H(\xi)_{-}}{\left(\xi-i \beta^{\prime}\right)_{-}^{1 / 2} \mathrm{M}\left(\xi / \beta^{\prime}\right)_{-}} \\
& =\frac{\left(\xi+i \beta^{\prime}\right)_{+}^{1 / 2} p(\xi)_{+}}{L\left(\xi / \beta^{\prime}\right)_{+}}-\left\{\frac{1}{i \xi}\left[\frac{\left(\xi+\beta^{\prime}\right)^{1 / 2}}{L\left(\xi / \beta^{\prime}\right)_{+}}-\frac{\left(i \beta^{\prime}\right)^{1 / 2}}{L(0)_{+}}\right]\right\}_{+}=0 .
\end{aligned}
$$

Fourier inversion gives

$$
H(z)=\frac{-1}{2 \pi\left(1+\lambda^{*}\right)\left(i \beta^{\prime}\right)^{1 / 2}} \int_{-\infty}^{\infty} \frac{\exp (i \xi z)}{\xi}\left(\xi-i \beta^{\prime}\right)^{1 / 2}\left\{\exp \left[\frac{1}{2 \lambda^{*}} \int_{0}^{\xi / \beta^{\prime}} S_{-}(x) d x\right]\right\} d \xi .
$$

$H(z \rightarrow 0)$ is found by evaluating the integral (8.15) as $\xi \rightarrow \infty$ [11]. The argument of the exponential involves the sum of three integrals, conveniently taken (without any approximations) as 


$$
\begin{aligned}
& I_{1}^{\prime}=-\frac{\lambda^{*}}{\pi} \int_{0}^{\xi / \beta^{\prime}}\left\{\left(\xi_{1}^{\prime}-k\right)^{-1}\left[\frac{\pi}{2}-i \ln \left(k+\lambda^{*^{-1}}\right)\right]\right. \\
& \left.\quad+\left(\xi_{1}^{\prime}+k\right)^{-1}\left[\frac{\pi}{2}-i \ln \left(\lambda^{*^{-1}}-k\right)\right]\right\} d \xi_{1}^{\prime}, \\
& I_{2}^{\prime}=\frac{1}{2}\left(\int_{0}^{\infty} d \xi_{1}^{\prime}-\int_{\xi / \beta^{\prime}}^{\infty} d \xi_{1}^{\prime}\right)\left\{\left(\xi_{1}^{\prime 2}+1\right)^{-1 / 2}\left[\left(\xi_{1}^{\prime}-k\right)^{-1}+\left(\xi_{1}^{\prime}+k\right)^{-1}\right]\right\}, \\
& I_{3}^{\prime}=-\frac{i}{\pi}\left(\int_{0}^{\infty} d \xi_{1}^{\prime}-\int_{\xi / \beta^{\prime}}^{\infty} d \xi_{1}^{\prime}\right)\left\{\left[\left(\xi_{1}^{\prime}-k\right)^{-1}+\left(\xi_{1}^{\prime}+k\right)^{-1}\right]\left(\xi_{1}^{\prime 2}+1\right)^{-1 / 2}\right. \\
& \left.\cdot \ln \left[\xi_{1}^{\prime}+\left(\xi_{1}^{\prime 2}+1\right)^{1 / 2}\right]\right\} .
\end{aligned}
$$

These integrals are now evaluated for $\xi / \beta^{\prime} \gg k \gg 1$.

The definition of the logarithm involved in the evaluation of $I_{1}^{\prime}$ is implicit in the path shown in Fig. 5:

$$
\begin{aligned}
I_{1}^{\prime}=\lambda^{*}\left\{-2^{-1}\left[\ln \left(\xi^{2}-k^{2}\right)-\right.\right. & 2 \ln k-i \pi] \\
& +i \pi^{-1}\left[\ln \left(\xi^{\prime}-k\right)-\ln k-i \pi\right] \ln \left(k+\lambda^{*^{-1}}\right) \\
& \left.+i \pi^{-1}\left[\ln \left(\xi^{\prime}+k\right)-\ln k\right] \ln \left(\lambda^{*^{-1}}-k\right)\right\} .
\end{aligned}
$$

For $\xi^{\prime} \gg 1$

$$
I_{1}^{\prime} \sim \lambda^{*}\left\{-\ln \xi^{\prime}+\ln k+i(\pi / 2)+\ln \left(k+\lambda^{*-1}\right)+O\left(\xi^{\prime-1}\right)\right\} .
$$

The semi-infinite integral in $I_{2}^{\prime}$ is standard; the other integral is negligible for current calculation:

$$
I_{2}^{\prime}=\left(\lambda^{*} / 2\right)\left\{\ln \left[k\left(1-k \lambda^{*}\right) /\left(1-\lambda^{*}\right)\right]+\ln \left[k\left(1+k \lambda^{*}\right) /\left(1-\lambda^{*}\right)\right]-i \pi\right\}+O\left(\xi^{-1}\right) \text {. }
$$

For $I_{3}^{\prime}$ one lets $\xi_{1}^{\prime}=\sinh \sigma$ in the semi-infinite integral:

$$
I_{3}^{\prime}=-\pi^{-1} i\left\{2 \int_{0}^{\infty} \frac{\sigma \sinh \sigma}{\sinh ^{2} \frac{k^{2}}{\sigma}} d \sigma+2 \frac{\ln \left(\xi^{\prime}\right)}{\xi^{\prime}}+O\left(\xi^{\prime-1}\right)\right\} .
$$

Twice the semi-infinite integral is the full-infinite integral, a multiple of which is given by contour integration (see Fig. 6 for the path):

$$
\oint_{c} \frac{(\sigma-i \pi)^{2} \sinh \sigma}{\sinh ^{2} \sigma-k^{2}} d \sigma=-2 \pi i \int_{-\infty}^{\infty} \frac{x \sinh x}{\sinh ^{2} \frac{k^{2}}{x-1}} d x=2 \pi i \sum_{j=1}^{2} \text { (residues) }_{j} .
$$

If $\sinh \tau=k$, then the enclosed singularities are simple poles at $x=-\tau$ and $x=\tau+i \pi$. Thus

$$
I_{3}^{\prime}=-2^{-1} \lambda^{*} \pi i-\lambda^{*} \ln \left\{\left[1+\left(1-\lambda^{*^{2}}\right)^{1 / 2}\right] / \lambda^{*}\right\}-2 \pi^{-1} i\left[\left(\ln \xi^{\prime}\right) / \xi^{\prime}\right]+O\left(\xi^{\prime-1}\right) .
$$

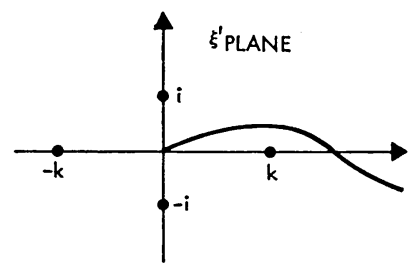

Fig. 5. Path of contour integral defined by $I_{1}^{\prime}$. 


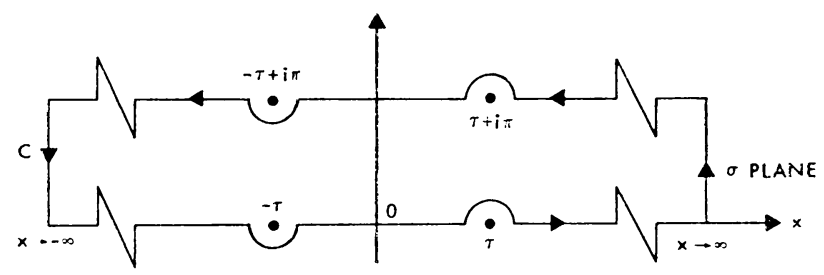

Fig. 6. The contour $C$ for the integral of Eq. (8.23). The path is taken below the singularity at $\rightarrow \tau$ to preserve the evenness of the integral.

Substitution of Eqs. (8.20), (8.21), and (8.24) in Eq. (8.15) yields for $0<\lambda^{*} \ll 1$

$$
H(z) \simeq \frac{i}{2 \pi \lambda^{* 1 / 2}} \int_{-\infty}^{\infty}\left(\xi-i \beta^{\prime}\right)^{1 / 2} \xi^{-3 / 2} \exp \left[i \xi z-i \beta^{\prime}(\ln \xi) / \pi \lambda^{*} \xi\right] d \xi .
$$

Recalling that for $z \rightarrow 0$ the exponential is expanded for large $\xi$ :

$$
H(z) \sim \frac{i}{2 \pi \lambda^{*^{1 / 2}}}\left\{\int_{-\infty}^{\infty} \xi^{-1} \exp (i \xi z) d z-\frac{i \beta^{\prime}}{\pi \lambda^{*}} \int_{-\infty}^{\infty} \xi^{-2}(\ln \xi) \exp (i \xi z) d \xi\right\} .
$$

To evaluate the first integral one recalls $H(z)=0$ for $z<0$ :

$H(z) \sim-\frac{1}{\lambda^{* 1 / 2}}+\frac{\beta^{\prime}}{2 \pi^{2} \lambda^{*^{3 / 2}}}\left\{z \int_{-\infty}^{\infty} v^{-2} \ln v \exp (i v) d v-z \ln z \int_{-\infty}^{\infty} v^{-2} \exp (i v) d v\right\}$.

The first integral in brackets yields a constant related to Euler's constant and the second gives by integration by parts

$$
H(z \rightarrow 0) \sim-\lambda^{*-1 / 2}+\beta^{\prime} \pi^{-1} \lambda^{*-3 / 2} z \ln z+O(z) \quad 1 \gg \lambda^{*} \gg z \geq 0 .
$$

The singularities at $\xi=0, i \beta^{\prime}$ give $H(z \rightarrow \infty)$ [11] [see Eq. (8.15)]; again, $\lambda^{*} \rightarrow 0$ is studied. The exponential factor of the integrand is unity at $\xi=0$ and goes as $1-\pi^{-1} \lambda^{*} \ln \lambda^{*}+O\left(\lambda^{*}\right)$ at $\xi=i \beta$. From Eq. (8.15)

$$
H(z \rightarrow \infty) \sim-\left\{1+2^{-1} \pi^{-1 / 2}\left(\beta^{\prime} z\right)^{-3 / 2}\left[\exp \left(-\beta^{\prime} z\right)\right]\left[1-\pi^{-1} \lambda^{*} \ln \lambda^{*}\right]\right\} .
$$

If $\lambda^{*}=0$, Eq. (8.29) recovers the asymptotic behavior described by Eq. (8.10).

Numerical evaluation of Eq. (8.15) [probably for $z=O\left(\lambda^{*}\right)$ ] would characterize more precisely those $z$ for which the slip correction merges with the no-slip result, Eq. (8.4). Without such calculations the small correction (owing to slip) of the drag implied by Eq. (8.4) cannot be precisely stated.

Fig. 7 compares the shear coefficient distribution $c_{f}$ along the flat plate $[-H=$ $\left(c_{f} / 2 A\right)$ where $A$ is defined below Eq. (8.2)] according to the classical no-slip Carleman formula for a short plate [Eq. (8.4)] and the model for a semi-infinite plate without slip [Eq. (8.10)]. Fig. 8 gives, for the semi-infinite plate model Eq. (8.5), the shear coefficient expression accounting for slight slip proposed for the leading edge [Eq. (8.28)]; the formal shear-with-slip expression obtained for distances far from the leading edge [Eq. (8.29)]; and the no-slip expression for the model [Eq. (8.10)]. The asymptotic forms are, of course, extrapolated beyond their domains of spatial validity in Fig. 8.

The increase of shear with $z$ for small $z$ when slip is included is noteworthy. The Monte Carlo simulation of Bird, Broadwell, and Vogenitz does give a rapid increase to a peak in the shear, then a gradual decrease along a long plate at hypersonic Mach number. 
Because the current nonmonotonic-shear results hold in the opposite limit $\mathrm{M} \rightarrow 0$ and are carried out for too short a plate for continuum theory to be demonstrably valid, the similarity may be fortuitous. Relevant experimental results for the leading-edge shear are not known to the author.

9. Extension to order-unity Prandtl number; thermal slip. Wu [6] has relaxed the condition $\mathrm{Pr} \rightarrow \infty$ and has treated Eqs. (2.5)-(2.7) for planar two-dimensional flows for $\operatorname{Pr}=3 / 4 \gamma$. For both large and small Reynolds numbers he found no cross-coupling exists to lowest order in the fundamental solutions for point sources of heat and momentum. That is, because dissipation is neglected in the energy equation, a momentum source leads to a velocity response but no thermal response, and a heat source leads to a thermal response but not velocity response. Thus, the results derived here for $\operatorname{Pr} \rightarrow \infty$ for $\operatorname{Re} \gg 1$ and $\operatorname{Re} \ll 1$ also hold for $\operatorname{Pr}=3 / 4 \gamma$.

These results imply that the formulation for thermal slip according to the MaxwellSmoluchowski formula leads to integral equations for $\operatorname{Re} \gg 1$ and $\operatorname{Re} \ll 1$ just like those posed for the velocity slip [6]. Specifically

$$
1+\epsilon T^{\prime}(x, 0)=1+\epsilon \theta_{w}+\beta_{2} \epsilon\left|\frac{\partial T^{\prime}(x, 0)}{\partial y}\right|
$$

along the plate where $\theta_{w}$ is the temperature of the plate and $\beta_{2}=[2 \gamma(\gamma+1)][(2-\alpha) / \alpha]$ $(\mathrm{Kn} / \mathrm{Pr})$ where $\alpha$ is the thermal accommodation coefficient $(.01 \leq \alpha<1)$. Roughly $\beta_{2} \simeq \mathrm{Kn} / \alpha$ for many gas-surface interactions. For a short flat plate

$$
\theta_{w}+\frac{\beta_{2}}{2} f(x)=\int_{-\mathrm{Re}}^{\mathrm{Re}}\left[\alpha_{1}+\beta_{1} \ln |x-\xi|\right] f(\xi) d \xi, \quad|x| \leq \mathrm{Re}
$$

where for $\mathrm{M}=0, \alpha_{1}=-(3 / 8 \pi \gamma)\left[\log (3 / 16)+\gamma_{1}\right]$ and $\beta_{1}=-3 / 8 \pi \gamma$. For a long flat plate

$$
\theta_{w}+\frac{\beta_{2}}{2} f(x)=a_{1} \int_{0}^{x} \frac{f(\xi) d \xi}{(x-\xi)^{1 / 2}}
$$

where $a_{1}=(3 / \pi)^{1 / 2}(4 \gamma)^{-1}$. Here $f(x)$ is the thermal gradient normal to the plate; it gives the heat transfer to the plate. Expressed nondimensionally, this quantity is the Nusselt number.

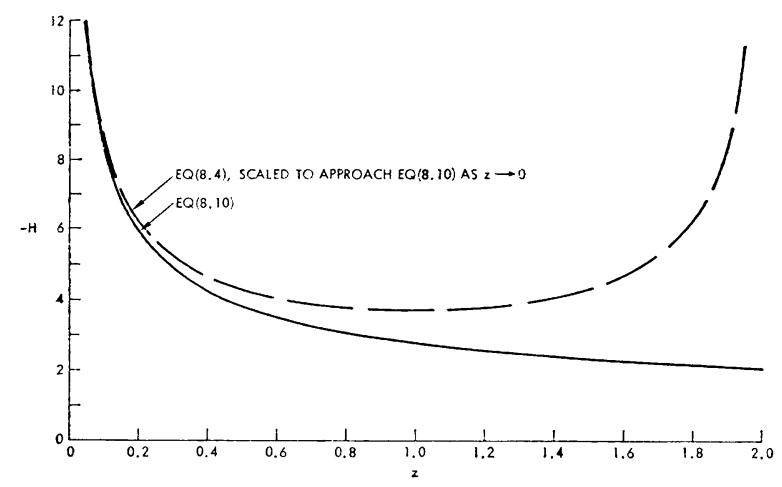

FIG. 7. The shear coefficient distribution along the flat plate $(M=0, \operatorname{Re}=0.1$, and $\lambda=0.1)$. 


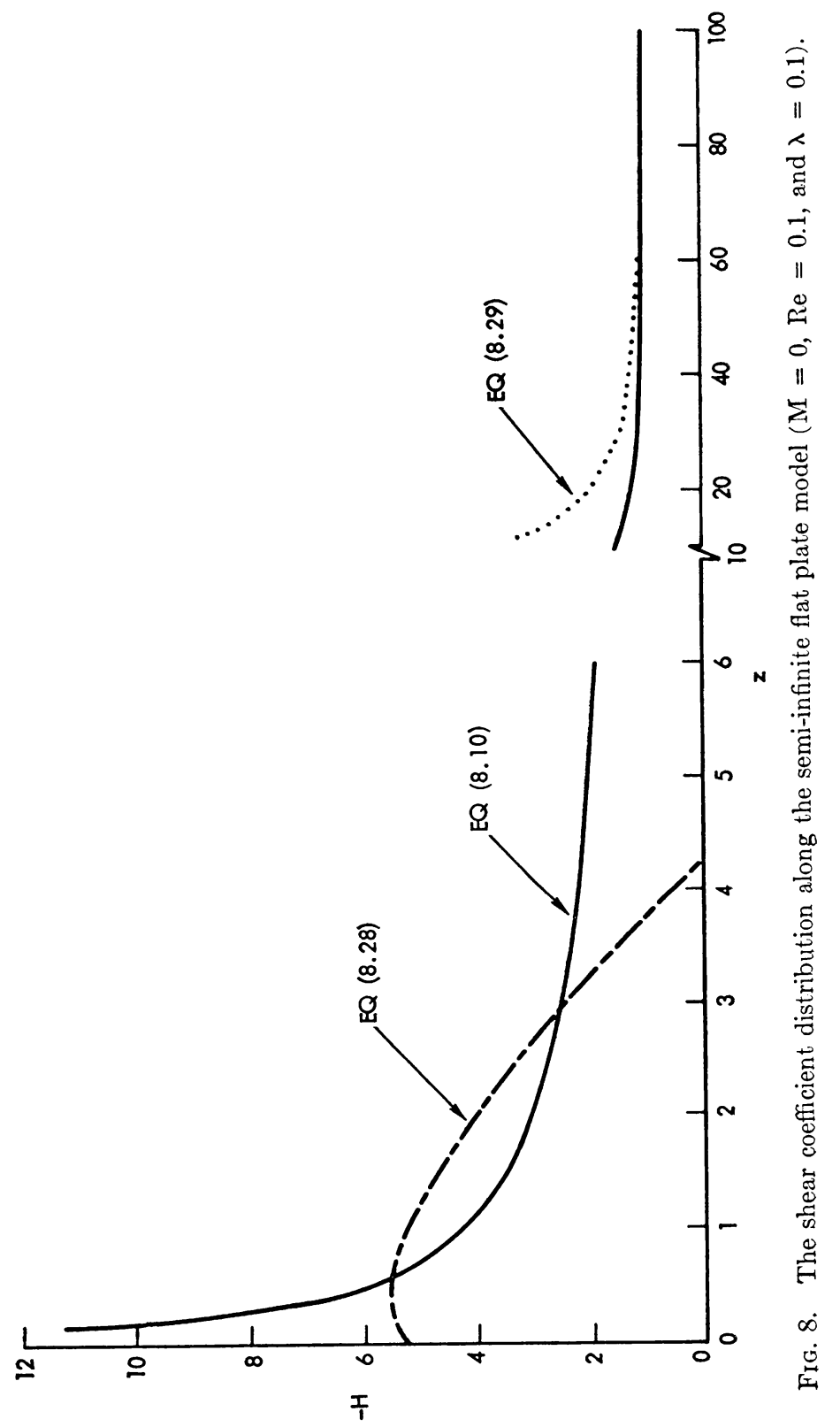


Acknowledgment. The author is deeply indebted for indispensable assistance from Professor P. A. Lagerstrom of Caltech throughout this work except for Sec. 8, which was carried out under guidance from Professor G. F. Carrier of Harvard University.

\section{References}

[1] C. W. Oseen, Neure Methoden und Ergebnisse in der Hyrdodynamik, Akad. Verlagsgesellschaft, Leipzig, 1927

[2] J. A. Laurmann, Slip flow over a short flat plate, Proc. First Internat. Sympos. on Rarefied Gas Dynamics, at Nice, ed. by F. M. Devienne, Pergamon, New York, 1960, pp. 293-316

[3] W. E. Olmstead and D. L. Hector, The lift and drag on a flat plate at low Reynolds number via variational methods, Quart. Appl. Math. 25, 415-422 (1968)

[4] P. A. Largerstrom, J. D. Cole, and L. Trilling, Problems in the theory of viscous compressible fluids, Caltech Guggenheim Aeronautical Laboratory Report, Pasadena, California, 1949

[5] M. Van Dyke, Impulsive motion of an infinite plate in a viscous compressible fluid, Z. angew. Math. Phys. 3, 343-353 (1952)

[6] T. Y. Wu, On problems of heat conduction in a compressible fluid, Caltech Ph.D. thesis, Pasadena, California, 1952

[7] —-, Anemometry of a heated flat plate, Proc. 1952 Heat Transfer and Fluid Mechanics Institute, Stanford Univ. Press, Stanford, 1952, pp. 139-158

[8] J. D. Cole and T. Y. Wu, Heat conduction in a compressible fluid, J. Appl. Mech. 19, 209-213 (1952)

[9] T. Y. Wu, Small perturbations in the unsteady flow of a compressible, viscous, and heat-conducting fluid, J. Math. and Phys. 35, 13-27 (1956)

[10] P. A. Lagerstrom, Laminar flow theory, in Theory of laminar flows, high speed aerodynamics and jet propulsion, Vol. 4, ed. by F. K. Moore, Princeton Univ. Press, Princeton, 1964, pp. 20-285

[11] G. F. Carrier, M. Krook, and C. E. Pcarson, Functions of a complex variable-theory and technique, McGraw-Hill, New York, 1966, Chaps. 6 and 8

[12] I. S. Gradshteyn and I. M. Ryzhik, Tables of integrals, series, and products, 4th ed., prepared by Yu. V. Geronimus and M. Yu. Tseytlin, transl. by A. Jeffrey, Academic Press, New York, 1965, p. 316

[13] G. A. Campbell and R. M. Foster, Fourier integrals for practical application, D. Van Nostrand, Princeton, N. J., 1948, p. 122, pair 912.2

[14] J. A. Laurmann, Linearized slip flow past a semi-infinite flat plate, J. Fluid Mech. 11, 82-96 (1961)

[15] - Structure of the boundary layer at the leading edge of a flat plate in hypersonic flip flow, AIAA J. 2, 1655-1657 (1964)

[16] S. Chapman and T. G. Cowling, The mathematical theory of nonuniform gases, 2nd ed., Cambridge Univ. Press, Cambridge, pp. 107-117

[17] S. G. Mikhlin, Integral equations, translated by A. H. Armstrong, Pergamon Press, New York, 1957, pp. 1-15

[18] H. Mirels, Estimate of slip effects on compressible laminar-boundary-layer skin friction, NACA TN 2609, Washington, D. C., 1952

[19] S. Bell, Studies of boundary-layer slip solutions and Alden's method for boundary-layer correction, University of California Institute of Engineering Research Report He-150-133, 1955

[20] J. D. Murray, Incompressible slip flow past a semi-infinite flat plate, J. Fluid Mech. 22, 463-469 (1965) 